\title{
Frequency locking near the gluing bifurcation: Spin-torque oscillator under periodic modulation of current
}

\author{
Michael A. Zaks*, Arkady Pikovsky \\ Institute of Physics and Astronomy, D-14476 Potsdam, Germany
}

\begin{abstract}
We consider entrainment by periodic force of limit cycles which are close to the homoclinic bifurcation. Taking as a physical example the nanoscale spin-torque oscillator in the LC circuit, we develop the general description of the situation in which the frequency of the stable periodic orbit in the autonomous system is highly sensitive to minor variations of the parameter, and derive explicit expressions for the strongly deformed borders of the resonance regions (Arnold tongues) in the parameter space of the problem. It turns out that proximity to homoclinic bifurcations hinders synchronization of spin-torque oscillators.
\end{abstract}

Keywords: Synchronization, homoclinic bifurcation, Arnold tongues, non-isochronous oscillators.

* Corresponding author. Tel.: +49 331 9775479, Fax +49 3319775947.

\section{Introduction}

Synchronization, commonly present in ensembles of interacting elements, is based on the tendency of coupled oscillators to adjust their characteristic timescales. Take a system in which several oscillators are synchronized, and perform a minor shift of one of the governing parameters. If the individual timescale of oscillations for every element is only slightly affected by this shift, it seems reasonable to expect that the collective state would sustain synchrony, up to small changes in its quantitative characteristics. If, in contrast, the timescale of one or several parts of the ensemble is highly sensitive to the parameter variation, adjustment to neighbors can get disrupted, and in the worst case synchronization breaks down. As a simple example, let every uncoupled unit perform the limit cycle dynamics. The situation when each limit cycle is robust and reacts to parameter variations only by modest shifts of frequency, is well understood (see e.g. [1, 2]: there, as a rule, synchronization is persistent. If, on the contrary, some of these limit cycles are fragile: stay on the verge of disappearance (via e.g. a saddle-node bifurcation), or if their frequencies strongly depend on the parameters (e.g. due to proximity to a homoclinic bifurcation), maintaining the synchrony can turn problematic.

In this paper, we take a closer look at the peculiarities of synchronization in the situation when the oscillating unit is close to the homoclinic bifurcation. As a physical object, we consider the spin-torque oscillator (STO) in the LC-circuit under the action of the periodic modulation of current. In recent years spin-torque oscillators have attracted broad interest as prospective nanoscale generators of electromagnetic microwaves in the gigahertz frequency range. A spin-torque oscillator is a device composed of two or more magnetic layers separated by non-magnetic spacers; electric current passes across one of such layers, becomes spin-polarized and exerts torque on the magnetic particles in the other layer, causing the high-frequency precession of the magnetization vector: spin-transfer effect [3]. The effect of giant magnetoresistance transforms this precession into microwaves. Since the field of a single spin-torque oscillator is weak, robust synchronous output of many such coupled units is desirable for applications. However, bringing ensembles of spin-torque oscillators to synchrony proved to be a difficult task.

Email addresses: mzaks@uni-potsdam.de (Michael A. Zaks*), pikovsky@uni-potsdam.de (Arkady Pikovsky) 
This seems to be related to the fact that spin-torque nano-oscillators are highly non-isochronous: the frequency of their oscillations is strongly dependent on the amplitude [4, 5]. By introducing an additional variable into the conventional Adler equation for the description of phase evolution, the authors of [6, 7] obtained explanations for a number of experimentally observable features, including the oscillatory transient dynamics which precedes the onset of the phase-locked state. Qualitative and numerical analysis of different configurations of the coupled STO gives evidence that in the operative range of relevant parameters each oscillator is, in a sense, close to homo- and heteroclinic bifurcations [8, 9, 10]. Below, we study the basic reasons for the difficulties of robust synchronization by considering sort of a minimal model: a single STO under the action of the periodic external force. It turns out that even the simplest entrainment: that by the periodic harmonic signal, is strongly hampered by proximity to homoclinics. Typical symmetry of the STO often ensures that the particular kind of homoclinic bifurcation: a so-called "gluing bifurcation" [11] in which stable periodic states coalesce and recombine, takes place. In the context of serially coupled arrays of STO gluing bifurcations were reported in [12]. Physically, closeness to a homoclinics means that minor changes of system parameters impel strong variations of characteristic times. Understandably, these variations hinder synchronization at a fixed frequency. Going beyond a particular model, we consider the general situation in which a periodic force acts upon a dynamical system which is close to the gluing bifurcation, and obtain expressions for the borders of the resonance regions (Arnold tongues) in the parameter space. These general theoretical predictions concern, as a particular case, the periodically forced STO.

The layout of the paper is as follows. In Sect. 2 we briefly delineate the mechanism which generates dynamics of the spin-torque oscillators. For the solitary spin-torque oscillator in the LC-circuit, we derive the governing equations and put them into the suitable form. In Sect. 3 we describe dynamics of that oscillator in absence of the external modulation. We show that increase of the constant current through the unit destabilizes the quiescent state and replaces it by small-scale oscillations of the magnetic moment. Further growth of the current leads to the formation of homoclinic orbits to the saddle equilibrium; in the large range of other parameters of the problem, this is a gluing bifurcation. Since in the course of transition the period of oscillations becomes infinite, entrainment of these oscillations by an external periodic force (which, in our case, is provided by modulations of the current in the circuit) is not quite trivial. In Sect. 4 we present a general formalism for the description of frequency locking near the gluing bifurcation. To this purpose, we construct the mapping which interrelates the coordinates of subsequent returns of the orbit into the vicinity of the equilibrium of the unperturbed system. Analysis of the mapping discloses the unusual shape of the Arnold tongues on the parameter plane of the problem. Entrained oscillations are stable in the region between the saddle-node, period-doubling and torus (Neimark-Sacker) bifurcations. Under low forcing frequencies, the distance between the two former bifurcations becomes exponentially small, and the entrainment, albeit formally present, can hardly be observed. The torus bifurcation, in contrast, is a resonant phenomenon, which occurs close to a certain particular frequency of forcing. Further, in the large parts of the parameter space, the entrained dynamics is (at least) bistable: two different kinds of periodic oscillations coexist. To illustrate these general findings, we return in Sect. 5 to the periodically forced spintorque oscillator and numerically determine the borders of Arnold tongues in the space of its parameters. In the end, along with the general discussion, we briefly outline the relation of the results to synchronization of two autonomous coupled STOs.

\section{Spin-torque oscillator: governing equations}

Consider a spin-torque oscillator in the LC-circuit (Fig 1). In the minimal configuration this is a stack of three layers: two magnetic ones and the nonmagnetic spacer between them. The thicker layer has constant magnetization, whereas the magnetization in the thinner one can freely rotate. Electric current passes through the thick magnetic layer, and, due to the interaction of electrons with magnetic field, becomes spin-polarized. Then, injected into a thin free magnetic layer, this polarized current induces precession of magnetization. Let us obtain the dynamical equations which govern this process. Our derivation and notation largely follow 13, 10]. Discarding the fine details of micromagnetic interactions inside the free layer, we restrict ourselves to the macroscopic description in the framework of the Landau-Lifshitz-GilbertSlonczewski magnetization equation. Within this approach, the unit vector $\vec{m}$ of magnetization in the free 


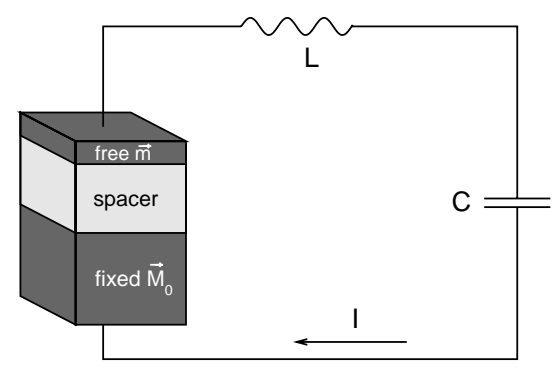

Figure 1: Spin-torque oscillator in the LC-circuit.

layer obeys equation of motion

$$
\frac{d \vec{m}}{d t}=-\gamma \vec{m} \times \vec{H}_{\mathrm{eff}}+\alpha \vec{m} \times \frac{d \vec{m}}{d t}+\gamma \bar{\beta} J \vec{m} \times\left(\vec{m} \times \vec{M}_{0}\right)
$$

where the last term, as demonstrated by Slonczewski [14], is responsible for the current-driven spin transfer. Here, $\gamma$ denotes the giromagnetic ratio, $\alpha$ is the Gilbert damping coefficient, and the effective Landau-Lifshitz field $\vec{H}_{\text {eff }}$ is a superposition of three components: the external magnetic field $\vec{H}_{a}$, the uniaxial anisotropy field $\vec{H}_{k}$ directed along the axis of easy magnetization, and the demagnetizing contribution $\vec{H}_{d z}$. In the spin-transfer term, $\vec{M}_{0}$ is the constant magnetization of the thick fixed layer, $\bar{\beta}$ characterizes the material properties of the free layer, and finally, $J$ is the instantaneous current through the circuit. Spin-transfer changes back and forth the magnetoresistance of the STO (see below), therefore $J=J(t)$ is, in general, timedependent, and Eq.(11), taken alone, is essentially non-autonomous. We align the $x$ - and $z$-axes along the directions of, respectively, the external field and the demagnetization field. Then, in Cartesian coordinates, Eq. (11) becomes

$$
\begin{aligned}
\mu \frac{d m_{x}}{d t}= & \left.H_{d z} m_{y} m_{z}+\alpha\left(\left(H_{a}+H_{k} m_{x}\right)\left(m_{y}^{2}+m_{z}^{2}\right)+H_{d z} m_{x} m_{z}^{2}\right)\right)-\bar{\beta} J M_{0}\left(m_{y}^{2}+m_{z}^{2}\right) \\
\mu \frac{d m_{y}}{d t}= & H_{d z} m_{z}\left(\alpha m_{y} m_{z}-m_{x}\right)-H_{k} m_{x}\left(\alpha m_{x} m_{y}+m_{z}\right) \\
& -H_{a}\left(\alpha m_{x} m_{y}+m_{z}\right)+\bar{\beta} J M_{0}\left(m_{x} m_{y}-\alpha m_{z}\right) \\
\mu \frac{d m_{z}}{d t}= & \left(H_{a}+H_{k} m_{x}\right)\left(m_{y}-\alpha m_{x} m_{z}\right)-\alpha H_{d z}\left(m_{x}^{2}+m_{y}^{2}\right) m_{z}+\bar{\beta} J M_{0}\left(m_{x} m_{z}+\alpha m_{y}\right)
\end{aligned}
$$

where the symbol $\mu$ denotes the combination $\left(1+\alpha^{2}\right) \gamma^{-1}$.

Since the rhs of Eq.(1) is orthogonal to the vector $\vec{m}$, its length is conserved, whereas direction varies in time. A characterization in terms of the spherical angles $\theta$ and $\varphi$ is suitable: $m_{x}=\sin \theta \cos \varphi, m_{y}=$ $\sin \theta \sin \varphi, m_{z}=\cos \theta$, and the equations turn into

$$
\begin{aligned}
\mu \frac{d \theta}{d t} & =\left(\alpha H_{a}-\bar{\beta} J M_{0}\right) \cos \theta \cos \varphi-\left(H_{a}+\alpha \bar{\beta} J M_{0}\right) \sin \varphi+\alpha S-T \\
\mu \sin \theta \frac{d \varphi}{d t} & =-\left(\alpha H_{a}-\bar{\beta} J M_{0}\right) \sin \varphi-\left(H_{a}+\alpha \bar{\beta} J M_{0}\right) \cos \varphi \cos \theta-S-\alpha T,
\end{aligned}
$$

where the symbols $S$ and $T$ denote, respectively, the combinations $\left(H_{d z}+H_{k} \cos ^{2} \varphi\right) \sin \theta \cos \theta \operatorname{and} H_{k} \sin \theta \sin \varphi \cos \varphi$.

Through the current $J$ these equations are coupled with dynamics of the LC-circuit. Electrodynamics of the latter obeys the Kirchhof equation

$$
L C \frac{d^{2} V}{d t^{2}}+V=R J=R\left(I-C \frac{d V}{D t}\right)
$$


where $V$ is the potential, $I$ is the ohmic current, $L$ and $C$ are, respectively, inductance and capacitance of the circuit, and resistance of the circuit $R$ is the sum of the ohmic resistance of the load and the resistance of the STO. The former is constant, but the latter, due to the effect of magnetoresistance, is time-dependent: as shown in [15], its value is a harmonic function of the instantaneous angle $\phi_{\vec{m}} \vec{M}_{0}$ between the magnetizations $\vec{m}$ and $\vec{M}_{0}$. The maximal value of resistance, $R_{p}$, is achieved in the case when both magnetizations are parallel; the minimal one, $R_{a p}$, corresponds to antiparallel magnetizations. In our configuration, $\vec{M}_{0}$ is directed along the $x$-axis, hence $\phi_{\vec{m} \vec{M}_{0}}=\cos ^{-1}(\sin \theta \cos \varphi)$. Accordingly,

$$
R\left(\phi_{\vec{m} \vec{M}_{0}}\right)=\frac{R_{p}+R_{a p}}{2}(1-\varepsilon \sin \theta \cos \varphi)
$$

where $\varepsilon$ denotes the ratio $\left(R_{p}-R_{a p}\right) /\left(R_{p}+R_{a p}\right)$.

On combining (3) with (4), denoting the product $\bar{\beta} M_{0}$ by $\beta$ and absorbing $\mu$ in the time units, we arrive at the system of four ODEs [10];

$$
\begin{aligned}
\frac{d \theta}{d t} & =\cos \theta \cos \varphi\left(\alpha H_{a}-\beta I(1-w)\right)-\sin \varphi\left(H_{a}+\alpha \beta I(1-w)\right)+\alpha S-T \\
\sin \theta \frac{d \varphi}{d t} & =-\sin \varphi\left(\alpha H_{a}-\beta I(1-w)\right)-\cos \varphi \cos \theta\left(H_{a}+\alpha \beta I(1-w)\right)-S-\alpha T \\
\frac{d u}{d t} & =\omega w \\
\frac{d w}{d t} & =\frac{\Omega_{0}^{2}}{\omega}((1-\varepsilon \sin \theta \cos \varphi)(1-w)-u)
\end{aligned}
$$

where the variables $u$ and $w$ are, respectively, rescaled $V$ and $\dot{V}$, the abbreviations $S$ and $T$ denote, as above, the combinations $\left(H_{d z}+H_{k} \cos ^{2} \varphi\right) \sin \theta \cos \theta$ and $H_{k} \sin \theta \sin \varphi \cos \varphi$, and the additional parameters which characterize the circuit are

$$
\omega=\frac{2\left(1+\alpha^{2}\right)}{\gamma\left(R_{p}+R_{a p}\right) C} \text { and } \Omega_{0}^{2}=\frac{\left(1+\alpha^{2}\right)^{2}}{\gamma^{2} L C} .
$$

These equations will be an object of our numerical investigations in the following sections. Recall that $I$ is the external current through the STO. Below, in Sect. 3 we treat $I$ as a constant; further, in Sect. 5 $I$ will be modulated around the constant value with frequency $\Omega$ and amplitude $\eta: I(t)=I_{0}+\eta \cos \Omega t$. In this way, the equations for both angular variables $\theta$ and $\varphi$ get affected by modulation.

Symmetry of the underlying physical problem finds reflection in the properties of the dynamical system. External magnetic field $\vec{H}_{a}$ singles out in the physical space a particular direction as well as the plane perpendicular to it. Accordingly, the Cartesian equations (3) are invariant with respect to the simultaneous change of sign of both components perpendicular to the field: $m_{y}$ and $m_{z}$. In terms of Eq. (6) this means invariance with respect to the transformation $(\theta \rightarrow \pi-\theta, \varphi \rightarrow-\varphi)$. This symmetry, in turn, influences the properties of the solutions.

\section{Dynamics of a single autonomous STO}

Consider an isolated spin-torque oscillator, governed by Eq. (6). Its phase space is a product of the sphere (angles $\theta$ and $\varphi$ ) and the plane (variables $u$ and $w$ ). Below, we briefly delineate basic changes in dynamics, invoked by the increase of the current $I$. We fix the values of other parameters at values taken in [10]:

$$
\alpha=0.01, \beta M_{0}=\frac{10}{3}, \omega=1, \varepsilon=0.3, H_{d z}=1.6, H_{k}=0.05, H_{a}=0.2 .
$$

Concerning the remaining coefficient $\Omega_{0}$, we start with the fixed value $\Omega_{0}=1 / 2$ used in [10], (there this coefficient was called $\Omega$ ), but later switch to $\Omega_{0}=3 / 2$; the reason will be explained below. 


\subsection{Equilibria and their stability}

A magnetic moment $\vec{m}$, set parallel to the external field $\vec{H}_{a}$, preserves its direction forever and stays constant; the off-field components will not be excited, and precession would not arise. There are two possibilities: $\vec{m}$ is directed either along $\vec{H}_{a}$ or in the opposite direction. In the chosen Cartesian coordinates, such states are characterized by $m_{x}= \pm 1$ whereas $m_{y}$ and $m_{z}$ vanish identically. Setting in Eq.(6) $\sin \varphi=0$, $\cos \theta= \pm 1$ yields these two equilibria which exist at all parameter values. Difference in the sign of the product $\cos \varphi \sin \theta$ between these states finds reflection in the difference of their stability properties.

\subsubsection{Quiescent state}

We start with the "natural" state of equilibrium in which the magnetic moment is aligned with the external field. Coordinates of this state in the phase space are:

$$
\theta=\frac{\pi}{2}, \varphi=0, u=1-\varepsilon, w=0 .
$$

Characteristic equation of its Jacobian is the product of two second order polynomials. The first one, independent of $\alpha, \beta, H_{a}, H_{k}, H_{d z}$ and the current $I$, yields two eigenvalues

$$
\lambda=\frac{\Omega_{0}}{2 \omega}\left(\Omega_{0}(\varepsilon-1) \pm \sqrt{\Omega_{0}^{2}(\varepsilon-1)^{2}-4 \omega^{2}}\right)
$$

For $\Omega_{0}<2 \omega /|\varepsilon-1|$ the latter are complex, and, since $\varepsilon<1$, possess negative real parts. The second factor is

$$
\lambda^{2}+2 \lambda\left(\alpha\left(H_{a}+H_{k}+\frac{H_{d z}}{2}\right)-\beta I\right)+\left(1+\alpha^{2}\right)\left(\beta^{2} I^{2}+\left(H_{a}+H_{k}\right)\left(H_{a}+H_{k}+H_{d z}\right)\right) .
$$

Since the last term is positive, these two eigenvalues cannot have opposite signs. If the current $I$ is absent or sufficiently weak, the equilibrium is stable; it gets destabilized in the Hopf bifurcation at

$$
I=I_{H}=\frac{\alpha}{\beta}\left(H_{a}+H_{k}+\frac{H_{d z}}{2}\right) .
$$

For the employed parameter values, this $\omega-, \Omega_{0^{-}}$and $\varepsilon$-independent threshold lies at $I_{H}=0.00315$.

\subsubsection{Saddle equilibrium}

Another equibrium state corresponds to the situation where the magnetic moment is directed opposite to the external field. It is natural to expect that such a state would be unstable; however, this is not necessarily the case. For this steady state with coordinate values

$$
\theta=\frac{\pi}{2}, \varphi=\pi, u=1+\varepsilon, w=0,
$$

the characteristic equation consists of two factors as well. The first one is the polynomial

$$
\lambda^{2}+\lambda \frac{\Omega_{0}^{2}}{\omega}(1+\varepsilon)+\Omega_{0}^{2}
$$

whose roots always possess negative real parts. For $\Omega_{0}>2 \omega /(\varepsilon+1)$ the roots are real; otherwise they are complex. Notably, the corresponding eigenvectors in the phase space are orthogonal to the plane of angular variables $\theta$ and $\varphi$.

The second factor of the characteristic equation is

$$
\lambda^{2}+2 \lambda\left(\beta I+\alpha\left(H_{k}-H_{a}+\frac{H_{d z}}{2}\right)\right)+\left(1+\alpha^{2}\right)\left(\beta^{2} I^{2}+\left(H_{k}-H_{a}\right)\left(H_{k}-H_{a}+H_{d z}\right)\right) .
$$

For $I<I_{s t}=\frac{1}{\beta} \sqrt{\left(H_{a}-H_{k}\right)\left(H_{k}-H_{a}+H_{d z}\right)}$, the last term is negative, and the steady state is a saddle. At $I=I_{s t}$ it gets stabilized in the course of the pitchfork bifurcation. 
For the employed values of $\beta, H_{a}, H_{k}$ and $H_{d z}, I_{s t}=0.1399$, and by far exceeds $I_{H}$. However, through variation of parameters (e.g. increase of $\alpha$, or, conversely, choosing close values of $H_{a}$ and $H_{k}$ ) the regions of stability of the two steady states can be made closer and even brought to an overlap.

In the case when the equilibrium (12) is unstable, the eigenvector which corresponds to the positive eigenvalue lies in the plane of coordinates $\theta$ and $\varphi$. Hence, the coordinate transformation $(\theta \rightarrow \pi-\theta, \varphi \rightarrow$ $-\varphi$ ) interchanges two components of the unstable manifold.

\subsubsection{Further steady states}

Besides (8) and (12), equations (6) possess other steady solutions. Two of them, in absence of external current $I$, are characterized by $m_{x}=-H_{a} /\left(H_{d z}+H_{k}\right), m_{y}=0, m_{z}= \pm \sqrt{1-m_{x}^{2}}, u=1-\varepsilon m_{x}$; explicit expressions for their coordinates at $I \neq 0$ are too long to be quoted here. At the employed values of the parameters the corresponding fixed points are unstable foci located in the polar regions of the sphere spanned by $\theta$ and $\varphi$. They do not play immediate role in the dynamics, but are helpful in classifying the periodic solutions of Eq. (6).

Outside the considered parameter range, further steady solutions may exist, e.g. symmetric branches born from (12) at $I=I_{s t}$; they are not relevant for the dynamics that we describe below.

\subsection{Peculiarities of homoclinic bifurcations}

The supercritical Hopf bifurcation at $I_{H}$ gives birth to the small-scale periodic oscillations of the magnetic moment. When the current $I$ is further increased, the amplitude of oscillations, as well as their temporal period grow, the corresponding phase portrait approaches the saddle point, and at a certain critical value $I=I_{\text {hom }}$ it gets captured by the unstable manifold of the saddle: the homoclinic bifurcation takes place. This event marks the transition from oscillations with bounded $(<2 \pi)$ amplitude with respect to $\varphi$ to unbounded rotations along this angular variable. Although this transition produces neither a topological change in the type of the closed phase trajectory on the sphere spanned by $\varphi$ and $\theta$, nor a noticeable change in the characteristics of the relevant physical observable $\left(x\right.$-component of the magnetic moment $\left.m_{x}\right)$, below for clarity we distinguish the "pre-homoclinic" $\varphi$-oscillations from the "post-homoclinic" $\varphi$-rotations.

Distinction between these two kinds of periodic solutions can be conveniently expressed in terms of location of the corresponding phase curves with respect to the fixed points on the surface of the sphere. Altogether, at $I>I_{H}$ there are four steady states: three unstable focus points and a saddle. A closed phase curve divides the surface of the sphere into two parts: the part with a single equilibrium of the focus type and the part with three remaining equilibria. We say that the single equilibrium is "encircled" by the curve. It is straightforward to see (cf Fig. 2) that the closed curve of $\varphi$-oscillations encircles the steady state (8) whereas the closed curve of $\varphi$-rotations encircles one of the near-polar foci. In this sense, oscillations characterize precession around the axis of the applied magnetic field whereas rotations correspond to precession with the axis close to the direction of demagnetization.
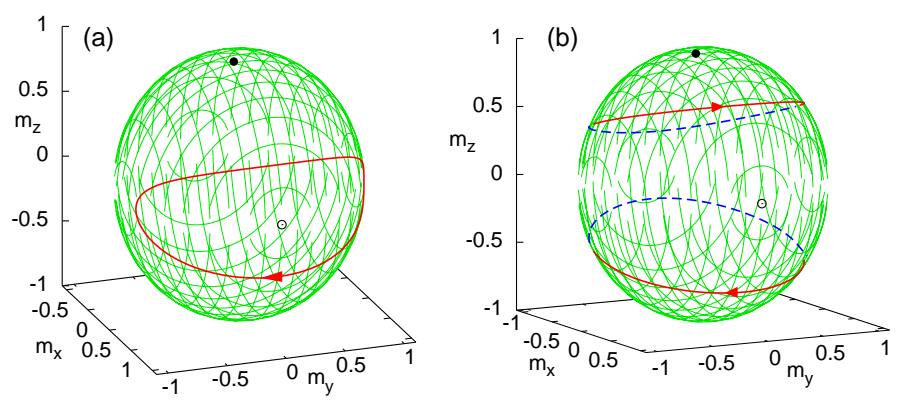

Figure 2: Closed orbits of Eq. (6) on the spherical surface. (a) $\varphi$-oscillations $(I=0.0035) ;(b): \varphi$-rotations $(I=0.008)$. Empty circle: equilibrium (8). Filled circle: unstable steady state in the polar regions. 
Symmetry of equations transforms each component of the unstable manifold of the saddle point into the other component. Therefore, homoclinic orbits occur in symmetric pairs: as a result, homoclinic bifurcations are accompanied in the phase space not by birth/destruction of closed orbits, as in the conventional homoclinic setup, but by their recombination ("gluing"), so that in the parameter space closed trajectories exist both before and after homoclinicity. The consequences of formation and breakup of homoclinic orbits depend on the type and relative magnitudes of the eigenvalues of the saddle. As proven in the seminal papers of Shilnikov [16, 17], in the situation when the Jacobian at the saddle point has a single positive eigenvalue $\lambda_{+}$, the decisive part is played by the $\operatorname{sum} \lambda_{+}+\operatorname{Re}\left(\lambda_{-}\right)$where $\lambda_{-}$is the eigenvalue with the closest to zero negative real part. If this sum (often called the "saddle quantity") is negative - in other words, if in the vicinity of the saddle contraction prevails over expansion, - the breakup of the homoclinic trajectory leaves in the phase space the unique stable periodic orbit. If the saddle quantity is positive, the periodic orbit is unstable. Moreover, if in the latter case $\lambda_{-}$is complex, the system possesses a countable set of unstable periodic orbits which, in many situations, serves as an indicator of chaotic dynamics.

Let us start with the case of $\Omega_{0}=1 / 2$. The $I$-independent complex eigenvalues from Eq. (13) equal $-0.1625 \pm 0.4729 \mathrm{i}$. In the parameter range $0.005<I<0.006$ where the homoclinic bifurcation is encountered, the real eigenvalues from (14) are $\approx 0.44$ and $\approx-0.49$. Accordingly, the steady state is a saddle-focus: the leading direction on its stable manifold corresponds to complex eigenvalues. Remarkably, since this direction is orthogonal to the plane of $\phi$ and $\theta$, the oscillatory stage in the approach to the steady state is almost invisible from the point of view of angular variables, as well as upon a stereographic projection. The fine structure, hardly visible in stereographic coordinates (left panel of Fig. 3) can be resolved with a blowup (middle panel), but is well recognizable in terms of the variable $u$ (right panel).
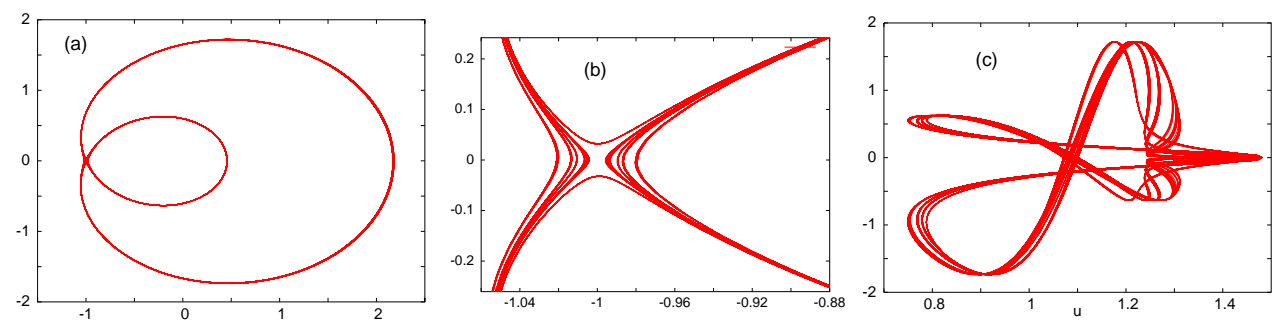

Figure 3: Projections of attractor at $\Omega_{0}=0.5, I=0.00568$, other parameters see (7). (a),(b): stereographic coordinates, $\cos \varphi \tan ((\pi-2 \theta) / 4)$ vs. $\sin \varphi \tan ((\pi-2 \theta) / 4)$. (c): $u$ vs. $\sin \varphi \tan ((\pi-2 \theta) / 4)$.

Since the repulsion along the unstable manifold is faster than the contraction along the leading direction of the stable one, the saddle quantity is positive, and existence of the pair of homoclinic trajectories implies presence of countably many unstable periodic orbits, horseshoes etc. Moreover, since the sum of three leading eigenvalues is positive, there should be no stable periodic solutions in the vicinity of homoclinic orbits [18].

Apparently, the stable periodic solution with bounded $\varphi$, born in the Hopf bifurcation and numerically observed until $I=0.005688$, does not directly participate in the homoclinic bifurcation. It comes reasonably close to the saddle point, so that its period grows from 27.045 at $I=0.005$ to 39.885 at $I=0.005688$, but disappears in the saddle-node bifurcation.

Tracking the unstable manifold of the saddle point indicates that homoclinics occurs slightly before that event, at $I=0.005624 \ldots$ The (first) homoclinic bifurcation and the disappearance of the stable limit cycle with bounded oscillations of $\varphi$ do not yet signify the transition to monotonic $\varphi$-rotations: immediately after it, (e.g. at $I=0.00567)$ clockwise and counterclockwise rotations alternate in a seemingly irregular way, as shown in the left panel of Fig. 4. Further from the bifurcation point (e.g. at $I=0.00568$ ) the trajectory makes a few consecutive rotations clockwise, followed by the same number of counterclockwise rotations, so that the angle $\varphi$ remains confined. Finally, starting from $I \approx 0.0057$, the monotonic drift of $\varphi$ begins.

Altogether, complicated dynamics is restricted to a small parameter range: $0.0055<I<0.0058$; outside it, attractors are periodic and have simple structure. Below, we do not elaborate on the aspects of chaotic dynamics. On the contrary, we would like to avoid complications associated to presence of countably many 

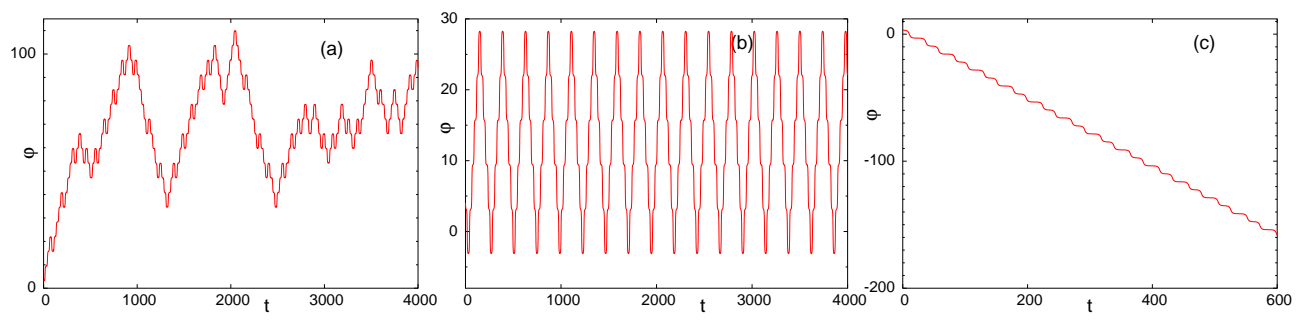

Figure 4: Temporal evolution of $\varphi$ close to homoclinics. $\Omega_{0}=0.5$, other parameters as in (7). (a): $I=0.00567$; (b) $I=0.00568$; (c) $I=0.0057$.

periodic orbits in the phase space and to deal with the evolution of just one periodic solution. For this purpose, at the event of homoclinic bifurcation both leading eigenvalues of the saddle state should be real. This can be achieved e.g. by varying the parameter $\Omega_{0}$ which influences the complex eigenvalues but leaves the real ones intact. When $\Omega_{0}$ is increased beyond $\approx 0.62$, contraction along the stable manifold becomes less than twice as fast as contraction along the stable one, hence close to homoclinicity stable periodic orbits should be dense in the parameter space [18]. Above $\Omega_{0} \approx 0.83$ the absolute value of the smallest negative real part of the eigenvalue exceeds the positive eigenvalue: the saddle quantity turns negative, and the homoclinic bifurcation gives rise to stable periodic solutions. Further on, with $\Omega_{0}$ above $\approx 0.85$, the smallest negative real part belongs not to the complex pair but to the real negative eigenvalue which corresponds to angular variables. Hence, the saddle-focus turns into the saddle point, with all usual implications for homoclinic bifurcations. In contrast to the case of the saddle-focus with its intermediate large scale oscillations, the homoclinic bifurcation with the saddle configuration immediately leads to monotonic evolution of $\varphi$. Since the leading direction on the stable manifold belongs to the plane of angular variables, two components of the unstable manifold approach the saddle from the opposite directions (so-called number eight configuration, opposed to the butterfly, familiar from the pictures of the homoclinic bifurcation in the Lorenz equations).

It is convenient to view the transformation of the trajectory in terms of the variable $\varphi$ unwrapped onto the line: the homoclinic orbit turns into the saddle connection between two $2 \pi$-shifted replicas of the saddle point (left panel of Fig. 5). Take a segment of the attracting trajectory which departs from the saddle along, say, its right separatrix, and therefore approaches the next (shifted) saddle from the right. Before the bifurcation, it turns back and returns to its initial position along the left separatrix, producing a closed trajectory. In contrast, after the bifurcation the same trajectory always passes the saddles "from the right", ensuring unbounded growth of $\varphi$ : the clockwise $\varphi$-rotation. Symmetric counterpart of this orbit describes the counterclockwise rotation. Up to non-conservation of energy, qualitatively this is the same heteroclinicsmediated transition from oscillations to rotations as in the classical nonlinear pendulum $\ddot{\varphi}+\sin \varphi=0$.

Finally, for $\Omega_{0}>2 \omega /(\varepsilon+1)=1.538$, all eigenvalues of the saddle steady state become real, without visible consequences for dynamics.

Variation of $\Omega_{0}$ does not affect the threshold $I_{H}$ of the onset of oscillations. A bit surprisingly, it also almost does not affect the value of $I$ which corresponds to the homoclinic bifurcation: for the configuration with $\Omega_{0}=0.5$ the latter occurs at $I=0.0056244 \ldots$ whereas for $\Omega_{0}=1.6$ it takes place at $I=0.0057098 \ldots$.

To ensure the uniqueness of the periodic orbit which participates in the homoclinic bifurcation, below we use the parameter value $\Omega_{0}=1.5$. When the values of the other parameters are fixed according to (7), the homoclinic bifurcation occurs at $I_{\text {hom }}=0.0056929063$. Close to the bifurcation, the period of the orbit logarithmically diverges (central panel of Fig. (5): $T \sim-A_{\mathrm{o}, \mathrm{r}} \log \left|I-I_{\text {hom }}\right|$. As seen on the left panel, during each oscillatory cycle the trajectory twice hovers in the neighborhood of the saddle point, whereas a cycle of $\varphi$-rotations includes only one passage close to the saddle. Therefore, the prefactor $A_{o}$ on the side of oscillations is twice as large as the prefactor $A_{r}$ on the side of rotations (right panel). Non-monotonic dependence of the period on $I$ leads to the remarkable consequence for the case of periodic driving: for a given value of the driving frequency, there are two ranges of $I$ where phase locking should be encountered.

We expect that strong variation of period within a small parameter interval should affect the sensitivity of oscillations to the external periodic signal. 

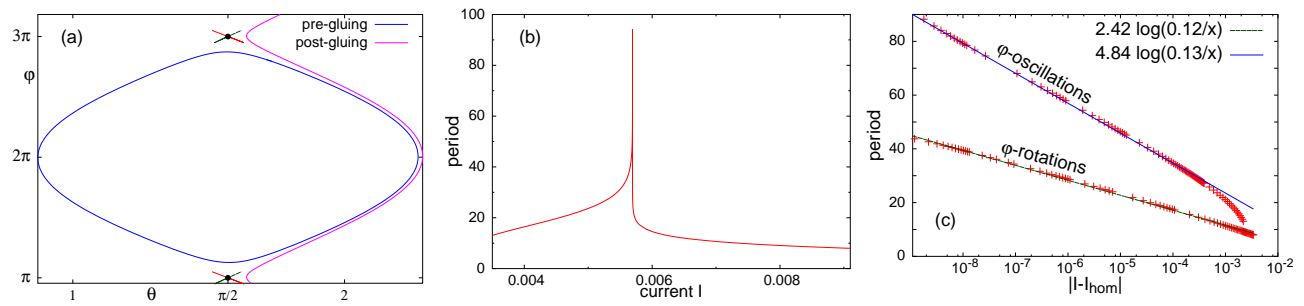

Figure 5: Gluing bifurcation. (a): transformation of the attracting orbit, (b),(c): parameter dependence of period of oscillations close to the bifurcation point.

\section{Return mapping and basic bifurcations in the case of external modulation}

Now we introduce periodic modulation of the current in Eq. (6) $: I(t)=I_{0}+\eta \cos \Omega t$. For simulations we take the frequency $\Omega$ which is of the order of the frequency of self-sustained oscillations in the autonomous system, as well as the values of $I_{0}$ above the threshold $I_{H}$, and the values of amplitude $\eta$ which do not exceed $I_{0}$. Numerical integration shows that phase portraits of oscillatory states include segments of trajectories which pass relatively close to the location of the saddle equilibrium in the autonomous system.

Let us construct and analyze a simplified mapping which interrelates the coordinates of subsequent returns into this region. We restrict ourselves to the case when in the spectrum of the saddle, the eigenvalue $\lambda_{-}$with the smallest negative real part is real; in Eq.(6) this corresponds to $\Omega_{0}>0.85$.

\subsection{Autonomous flow}

To start with, we review the return mapping in the autonomous situation. We do not discriminate between an autonomous saddle point (say, $\theta=-\pi / 2, \varphi=0$ ), and all its replicas shifted by $2 \pi n(n=$ $\pm 1, \pm 2, \ldots)$ along $\varphi$. However, in order to capture the difference between $\varphi$-oscillations and $\varphi$-rotations, we need to consider the region of phase space with two such replicas (see Fig 6).

We parameterize directions along the unstable and stable manifolds of the saddle point by local coordinates $x$ and $z$, respectively 1 . Two components of the unstable manifold $W^{u}$ (separatrices) are transformed into each other by the simultaneous change of sign of $x$ and $z$. The same transformation maps every orbit of the system either onto itself, as in the case of periodic oscillations, or onto its symmetric image (e.g. clockwise and counterclockwise rotations).

Near the saddle point, the equations of motion are

$$
d x / d t=\lambda_{+} x+\text { h.o.t. }, \quad d z / d t=\lambda_{-} z+\text { h.o.t. }
$$

where $\lambda_{+}$and $\lambda_{-}$are, respectively, the positive and the closest to zero negative eigenvalues of the Jacobian at the saddle. The usual formalism divides the phase space into the "local" part near the saddle (shown as gray polygon in Fig, 6, and the "global' region far from the fixed point(s) where the trajectories stay close to the separatrices. Trajectories enter the local region by crossing either of two lines $A, B$, transversal to the stable manifold of the saddle, and exit by crossing either of two lines $G$, transversal to its unstable manifold. These borders are taken sufficiently close to the saddle, so that the flow in the local region can be viewed as linear. The Poincaré sections are taken at the entrance to the local region, on both sides of the saddle, at small distances $\pm z_{s}$ from its local unstable manifold. An orbit which starts from either of the secants $A, B$ with a positive (negative) value of $x$, departs from the saddle along the "right" ("left") separatrix, and arrives to the region of the saddle replica, shifted with respect to $\varphi$ by $2 \pi(-2 \pi)$. Accordingly, we divide each secant into the left and right parts $A_{l}$ and $A_{r}$, respectively $B_{l}$ and $B_{r}$. A section is characterized by the coordinates $x_{i}$ and $z_{i}$ of the intersection point; in fact, for the latter only the sign matters. In the global region, trajectories stay close to the separatrices of the saddle.

\footnotetext{
${ }^{1}$ this notation is not related to orientation of the magnetization vector from Eq. (1).
} 

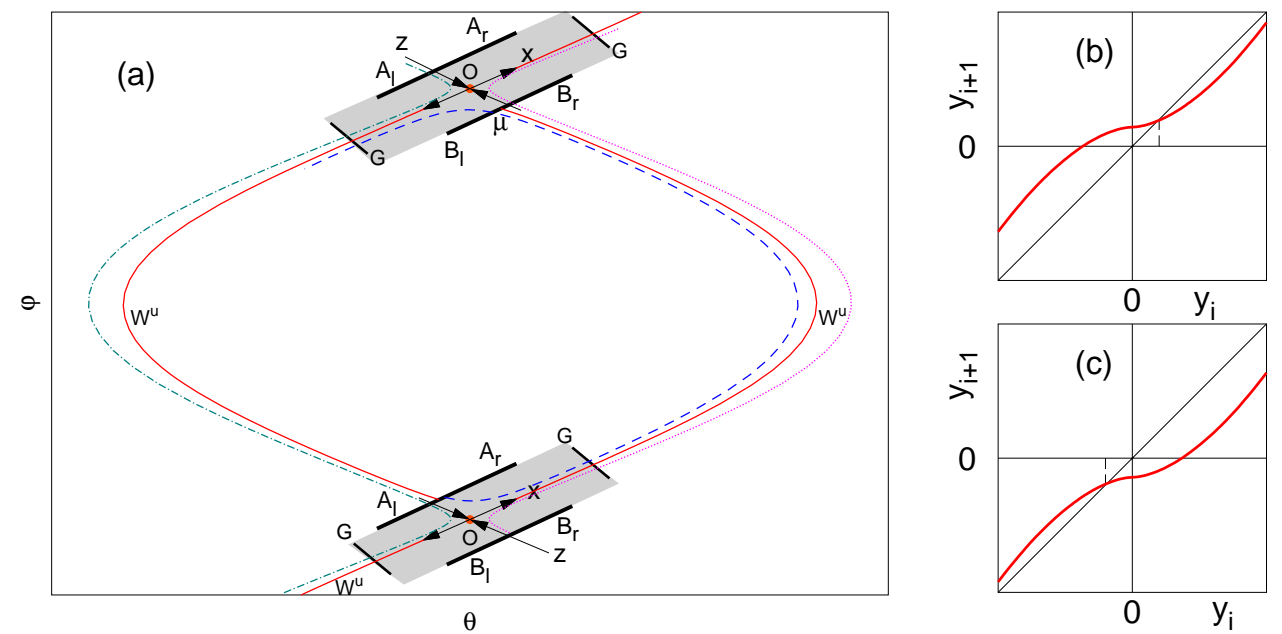

Figure 6: Return mapping near the saddle point. (a): Construction of the mapping. Gray domains: regions of local (linearized) dynamics. $O$ : copies of the saddle point; red curves $W^{u}$ : components of the unstable manifold; $A_{l, r}, B_{l, r}$ : Poincaré secants, transversal to the stable manifold; $G$ : secants, transversal to $W^{u}$; dashed and dotted lines: typical phase trajectories passing through the vicinities of the saddle.

(b),(c): One-dimensional map [18) with $\nu>1$ at, respectively, negative and positive $\mu$.

This approach allows to interrelate the coordinates of consecutive arrivals on Poincaré secants; in the lowest relevant order these relations read

$$
x_{i+1}=\mu+a x_{i}^{\nu} \operatorname{sign}\left(z_{i}\right), z_{i+1}=-z_{s}
$$

for orbits with positive $x_{i}$ which move along the right separatrix, and

$$
x_{i+1}=-\mu-a\left|x_{i}\right|^{\nu} \operatorname{sign}\left(z_{i}\right), \quad z_{i+1}=z_{s}
$$

for orbits with negative $x_{i}$ which depart along the left separatrix. Here, $\mu$ is the $x$-coordinate of the first intersection of the right separatrix with the Poincaré secant $z=-z_{s}$; the value $\mu=0$ corresponds to formation of the homoclinic trajectory. Variation of the parameter $\mu$ roughly reflects variation of the current $I_{0}$ in the original equations. Further, the saddle index $\nu=\left|\lambda_{-}\right| / \lambda_{+}$shows which process - contraction or expansion - prevails in the vicinity of the saddle point. According to the Shilnikov theory, values of $\nu$ between 0 and 1 ensure instability of periodic orbits, born from the breakup of the homoclinic trajectory. For $\nu>1$ such orbits are, on the contrary, stable; this corresponds to the relevant region of the parameter space of Eq.(6), where the value of $\nu$ lies between 1.6 and 1.7. Finally, the factor $a$ is the so-called "separatrix value"; its sign shows whether the mapping along the separatrix preserves orientation. Numerical simulation of Eq. ([6) indicates that in our situation (which is reminiscent of dynamics on the two-dimensional sphere) the mapping is orientable, hence $a$ is positive; below we set $a=1$ (at $\nu \neq 1$ this is achieved by rescaling the units of $x$ ).

To ensure validity of the mapping, the system should stay sufficiently close to homoclinicity, and the considered trajectories should be selected not too far from the separatrices. Expressed in terms of $\mu$ and $y$, this reads $|\mu| \ll(\nu-1) \nu^{\nu /(1-\nu)}$ and $|y|<\nu^{1 /(1-\nu)}$.

To unify two mappings (16) and (17), we introduce the auxiliary variable $y=x \operatorname{sign}(z)[20] ; y$ is invariant with respect to the simultaneous change of signs of $x$ and $z$. In terms of $y$, the return mapping reduces to

$$
y_{i+1}=-\mu+\left|y_{i}\right|^{\nu} \operatorname{sign}\left(y_{i}\right)
$$

Plots of the piecewise-smooth mapping (18) for $\mu<0$ and $\mu>0$ are shown in Fig 6 (b,c). At small negative values of $\mu$ the mapping possesses a stable fixed point which lies close to zero at $y>0$; at small 
positive values of $\mu$, the value of $y$ in the fixed point is negative. While interpreting this in terms of the original variables, we should keep in mind the assignment of signs in $y$. The fixed point with positive $y$ corresponds e.g. to passage from $A_{r}$ to $B_{l}$ in Fig. 6. since there the hitherto ascending trajectory turns down and returns to the lower saddle, the iteration of the mapping corresponds to the half of the oscillation of $\varphi$; the complete cycle of oscillation requires two iterations. In contrast, the fixed point with negative $y$ corresponds to the passage of the trajectory from $A_{r}$ upwards to $A_{r}$ or from $B_{l}$ downwards to $B_{l}$. With each new iteration this passage is repeated, hence the fixed point with negative $y$ describes the clockwise and the counterclockwise rotations of the variable $\varphi$. Since the mapping (18) is monotonic, there are no other local attractors. For completeness we also mention two distant unstable fixed points of the mapping, with large values of $|y|$ (at $\mu=0$ they lie at $y= \pm 1$ ); the point with positive $y$ may be vaguely associated with the equilibrium (8), destabilized in the course of the Hopf bifurcation.

\subsection{Return mapping under periodic forcing}

With this picture in mind, let us introduce a sufficiently weak periodic force acting on both leading variables. The forced equations of motion read

$$
\begin{aligned}
& d x / d t=\lambda_{+} x+A \cos \omega t+\text { h.o.t. } \\
& d z / d t=\lambda_{-} x+A \cos \omega t+\text { h.o.t. }
\end{aligned}
$$

When the nonlinear terms are discarded, the solutions are $x(t)=c_{1} \mathrm{e}^{\lambda_{+} t}+x_{p}(t)$ and $z(t)=c_{2} \mathrm{e}^{\lambda_{-} t}+z_{p}(t)$ where $x_{p}(t)$ and $z_{p}(t)$ are components of the unstable periodic solution

$$
x_{p}(t)=\frac{A}{\omega^{2}+\lambda_{+}^{2}}\left(\omega \cos \omega t-\lambda_{+} \sin \omega t\right), z_{p}(t)=\frac{A}{\omega^{2}+\lambda_{-}^{2}}\left(\omega \cos \omega t-\lambda_{-} \sin \omega t\right) .
$$

On rewriting the equations in the corotating reference frame by introducing the variables $\tilde{x} \equiv x-x_{p}(t)$ and $\tilde{z} \equiv z-z_{p}(t)$, they become

$$
d \tilde{x} / d t=\lambda_{+} \tilde{x}+F_{1}(\tilde{x}, \tilde{z}, t), \quad d \tilde{z} / d t=\lambda_{-} \tilde{z}+F_{2}(\tilde{x}, \tilde{z}, t)
$$

where $F_{1}$ and $F_{2}$ consist of nonlinear terms with explicit periodic dependence on $t$. In this sense, additive periodic forcing turns into periodic modulation of the parameter(s).

Equations (19) have an equilibrium point at the origin: $\tilde{x}=\tilde{z}=0$. For the derivation of the return mapping, the above formalism should be extended in order to account for the variability of the force at the moments of intersection with the Poincaré secants. As a coordinate characterizing the external forcing, it is convenient to take the phase $\Psi$ of the force; it obeys the equation $d \Psi / d t=\omega$. For the given orbit, let $\tau_{\text {loc }}$ and $\tau_{\mathrm{g}}$ be the times of passage of the trajectory through, respectively, the local and the global region. Then, the increment of the phase $\Psi$ between subsequent intersections equals $\omega\left(\tau_{\text {loc }}+\tau_{\mathrm{g}}\right)$. The value of $\tau_{\mathrm{g}}$, in general, depends on the values of $\tilde{z}$ and $\Psi$ at the exit from the local region; to start with, we neglect this dependence and treat $\tau_{\mathrm{g}}$ as a constant. The local passage time $\tau_{\text {loc }}$ depends on the place of entry to the local region; up to an additive constant (which can be absorbed into $\tau_{\mathrm{g}}$ or, by rescaling $x$, can be turned into zero), it equals $-\lambda_{+}^{-1} \log \left|\tilde{x}_{i}\right|$.

We expect that effect of the weak forcing upon the section coordinate $\tilde{x}_{i}$ can be represented as an additive modulation, proportional to the value of the force at the exit from the local region. In this way, we arrive at the mapping which relates the values of $y($ recall that $y=\tilde{x} \operatorname{sign}(\tilde{z}))$ and $\Psi$ at the moments of subsequent intersections with the Poincaré planes:

$$
\begin{aligned}
y_{i+1} & =-\mu+\left|y_{i}\right|^{\nu} \operatorname{sign}\left(y_{i}\right)+B \sin \left(\Psi_{i}-\omega \lambda_{+}^{-1} \log \left|y_{i}\right|\right) \\
\Psi_{i+1} & =\Psi_{i}+\omega\left(-\lambda_{+}^{-1} \log \left|y_{i}\right|+\tau_{\mathrm{g}}\right)
\end{aligned}
$$

where $B$ is proportional to the forcing amplitude $A$.

The mapping of this kind and its dynamics were described in [19]; there, the frequency of the forcing was not explicitly included in the analysis. In our setup, the driving frequency $\omega$ plays the crucial role, and 
we treat it as an independent parameter. A similar mapping was partially analyzed in [21] in the context of chaotic synchronization; there, the case of $\nu<1$ was considered, so that the corresponding periodic orbit in the autonomous system was unstable.

A periodic solution of the continuous system corresponds to the fixed point of the mapping (20): $y_{i+1}=y_{i}$ and $\Psi_{i+1}=\Psi_{i}+2 \pi$. The latter relation yields two values for the coordinate $y$ of the fixed point:

$$
y= \pm \exp \left(\lambda_{+}\left(\tau_{\mathrm{g}}-\frac{2 \pi}{\omega}\right)\right) .
$$

Recall that the fixed point with positive $y$ corresponds to the $\varphi$-oscillations whereas the fixed point with $y<0$ describes the rotational state. Each value of $y$, in its turn, leads to two values of the phase of the force $\Psi$ :

$$
\Psi=\omega \tau_{\mathrm{g}}+\left\{\begin{array}{c}
\arcsin \frac{\mu+y-|y|^{\nu} \operatorname{sign}(y)}{B} \\
\pi-\arcsin \frac{\mu+\left.y\right|^{\nu} \operatorname{sign}(y)}{B}
\end{array}\right.
$$

For the analysis of existence and stability of the fixed point we take more general functional dependencies:

$$
\begin{aligned}
y_{i+1} & =f\left(y_{i}\right)+B g\left(\Psi_{i}+\omega \tau_{\mathrm{loc}}\left(y_{i}\right)\right) \\
\Psi_{i+1} & =\Psi_{i}+\left(\tau_{\mathrm{loc}}\left(y_{i}\right)+\tau_{\mathrm{g}}\right) \omega
\end{aligned}
$$

using, along with the $2 \pi$-periodic function $g$, the characteristics of the autonomous system: the return mapping $f(y)$ and the passage time $\tau_{\text {loc }}(y)$.

The saddle-node bifurcation in this system takes place at

$$
g^{\prime}(\phi) \tau_{\mathrm{loc}}^{\prime}(y)=0 ;
$$

here and below $\phi$ is the abbreviation for the argument of $g$, and all derivatives are taken at the fixed point. Since the local passage time $\tau_{\text {loc }}(y)$ has no extrema, this condition reduces to $g^{\prime}(y)=0$.

The period-doubling bifurcation requires

$$
2+2 f^{\prime}(y)+B g^{\prime}(\phi) \omega \tau_{\mathrm{loc}}^{\prime}(y)=0 .
$$

Finally, the torus (Neimark-Sacker) bifurcation occurs at

$$
f^{\prime}(y)=1
$$

Substituting the particular dependencies $f, g$ and $\tau_{\text {loc }}(y)$, we obtain explicit expressions for the stability boundaries.

\subsubsection{Oscillations}

On the parameter plane of $\omega$ and $B$, the pair of fixed points (22) with positive $y$ exists above the curve of the saddle-node bifurcation

$$
B_{\mathrm{sn}}^{\mathrm{o}}(\omega)=\left|\mu+\mathrm{e}^{\lambda_{+}\left(\tau_{\mathrm{g}}-2 \pi / \omega\right)}-\mathrm{e}^{\nu \lambda_{+}\left(\tau_{\mathrm{g}}-2 \pi / \omega\right)}\right|
$$

which has the "infinitely flat" horizontal asymptote at $\omega \rightarrow 0$.

At $\mu<0$, when the oscillatory solution is present already in the autonomous system, the region of existence (Arnold tongue) opens up from the point at the abscissa $B=0$; the value of $\omega$ in this point equals the frequency $\omega_{\text {aut }}$ of autonomous oscillations. On the left boundary of the tongue,

$$
B_{\mathrm{sn}}^{\mathrm{o}}(\omega)=-\mu-\mathrm{e}^{\lambda_{+}\left(\tau_{\mathrm{g}}-2 \pi / \omega\right)}+\mathrm{e}^{\nu \lambda_{+}\left(\tau_{\mathrm{g}}-2 \pi / \omega\right)},
$$

at very small $\omega$ the asymptote is approached from below; note that two $\omega$-dependent terms contribute with different signs. 
In contrast, at $\mu>0$, when the oscillatory solution is absent in the autonomous system, the expression (24) is bounded away from zero at small and moderate values of $\omega$. Here, an oscillation can be excited by the periodic driving force, provided that the amplitude $B$ exceeds the frequency-dependent threshold value and, in any case, is larger than $\mu$.

Just above the saddle-node bifurcation, one of the newborn fixed points is stable (the second one is a saddle). The region of stability is bounded from above by the curve of the period-doubling bifurcation $B_{\text {pd }}(\omega)$. It is convenient to characterize this curve in terms of its elevation with respect to $B_{\mathrm{sn}}(\omega)$ :

$$
B_{\mathrm{pd}}^{2}(\omega)-B_{\mathrm{sn}}^{2}(\omega)=\frac{4 \lambda_{+}^{2} y^{2}}{\omega^{2}}\left(1+\nu|y|^{\nu-1}\right)^{2}
$$

Since $y$ is exponentially small with respect to $\omega$ (cf. Eq.(21) ), the stability region becomes very narrow and, practically, nearly vanishes at low values of the driving frequency. Remarkably, however, two bounding curves do not intersect at finite $\omega$.

It should be recalled that the fixed point of the mapping (20) with positive $y$ corresponds not to the whole cycle of oscillations, but to half of that cycle; in the flow, it describes symmetric phase curves which are mapped onto themselves by transformation $\varphi \rightarrow-\varphi, \theta \rightarrow \pi / 2-\theta$. Accordingly, the perioddoubling bifurcation in (20) will, in terms of the flow, be not the period-doubling, but the symmetry-breaking (pitchfork) bifurcation of the limit cycle.

The Neimark-Sacker bifurcation at which two Floquet multipliers cross from inside the unit circle, and the invariant curve of the mapping is born, turns out to be a resonant phenomenon: independently of the values of $\mu$ and $B$ (the latter should be large enough to ensure the existence of the fixed point), it occurs when the driving frequency equals

$$
\omega_{\mathrm{NS}}=\frac{2 \pi}{\tau_{\mathrm{g}}-\frac{\log \nu}{\lambda_{+}(1-\nu)}}
$$

For $\omega>\omega_{\mathrm{NS}}$, both periodic solutions, born in the saddle-node bifurcation at $B=B_{\mathrm{sn}}$, are unstable. The place where the Neimark-Sacker bifurcation branches off the saddle-node curve, lies on the right border of the Arnold tongue, at the highest point of that borden 2. Bifurcation diagrams for the fixed points of the
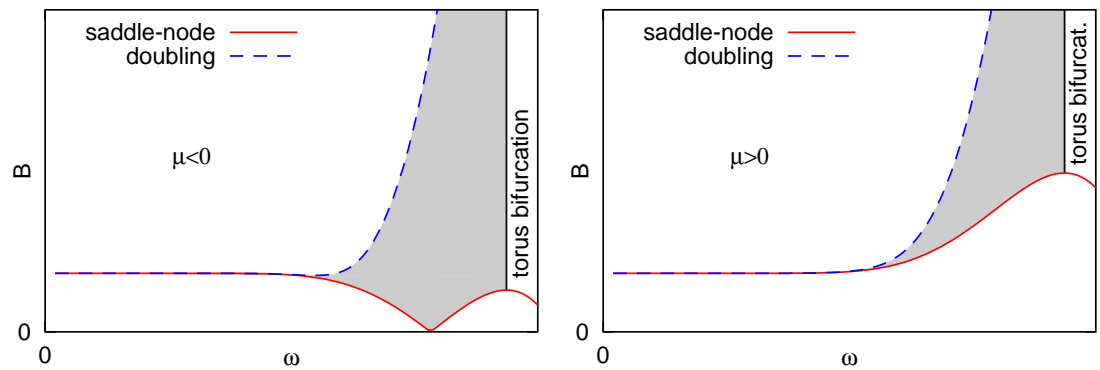

Figure 7: Bifurcation diagram for periodic $\varphi$-oscillations. Gray regions: existence of stable periodic state. Left panel: $\mu<0$ (autonomous system is pre-homoclinic). Right panel: $\mu>0$ (post-homoclinic situation).

mapping are shown in Fig. [7. Part of the diagram to the right from the Neimark-Sacker bifurcation is not relevant: it refers to bifurcations of the distant fixed point of the autonomous mapping (18) with non-small value of $y$.

\footnotetext{
${ }^{2}$ The fixed point implies $y=f(y)+B g\left(\Psi, \tau_{\text {loc }}(y)\right)$. Differentiation with respect to $\omega$ yields

$$
\frac{d B}{d \omega}=\frac{1-f^{\prime}(y)}{g\left(\Psi, \tau_{\mathrm{loc}}(y)\right)} \frac{\partial y}{\partial \omega}-\frac{(y-f(y)) g^{\prime}\left(\Psi, \tau_{\mathrm{loc}}(y)\right)}{g\left(\Psi, \tau_{\mathrm{loc}}(y)\right)^{2}}\left(\frac{\partial \Psi}{\partial \omega}+\tau_{\mathrm{loc}}^{\prime}(y) \frac{\partial y}{\partial \omega}\right)
$$
}

Since $g^{\prime}=0$ along the saddle-node curve, the last term vanishes there. The Neimark-Sacker condition $f^{\prime}(y)=1$ lets the first term vanish as well, hence the codimension-two event occurs at the extremum of the saddle-node curve. 


\subsubsection{Rotations}

For rotations, the Arnold tongue on the parameter plane is bounded by the line

$$
B_{\mathrm{sn}}^{\mathrm{r}}(\omega)=\left|\mu-\mathrm{e}^{\lambda_{+}\left(\tau_{\mathrm{g}}-2 \pi / \omega\right)}+\mathrm{e}^{\nu \lambda_{+}\left(\tau_{\mathrm{g}}-2 \pi / \omega\right)}\right|
$$

At positive values of $\mu$, when $\varphi$-rotations exist in the autonomous case as well, the Arnold tongue opens from the abscissa $B=0$. For $\mu<0$, the region of existence of rotational solutions is separated from the abscissa: without the force there are no rotations, and in order to excite them, the frequency-dependent threshold should be exceeded. Parts of the diagram to the right from the curve of the torus bifurcation refer to the unstable distant fixed point, fictitious from the point of view of the underlying flow.

Like in the case of oscillations, the region of stability of the fixed point is bounded from above by the curve of the period-doubling bifurcation which (again, like in that case) obeys Eq.(25) so that at low frequencies the range of stability is nearly absent. In contrast to the $\varphi$-oscillations, the period-doubling in the mapping describes the genuine period-doubling of the underlying flow.

The modulation instability of the periodic rotational solution occurs at the same value of frequency (26), as for the oscillations.

Remarkably, at low values of the driving frequency the lower borders of Arnold tongues for $\varphi$-oscillations and $\varphi$-rotations nearly coincide: the corresponding values of $B_{\mathrm{sn}}$ are separated by the exponentially small term $2 \mathrm{e}^{\lambda_{+}\left(\tau_{\mathrm{g}}-2 \pi / \omega\right)}$. At negative $\mu$, the threshold for oscillations lies slightly lower; at positive $\mu$, the opposite situation takes place. In its turn, this implies that the borders of period-doubling bifurcation for rotations and oscillations almost coincide as well.

The bifurcation diagram for periodic rotations is sketched in Fig 8 , When the same values of $|\mu|$ are used for oscillations and rotations, the panels of Fig 8 look like the interchanged panels of Fig 7
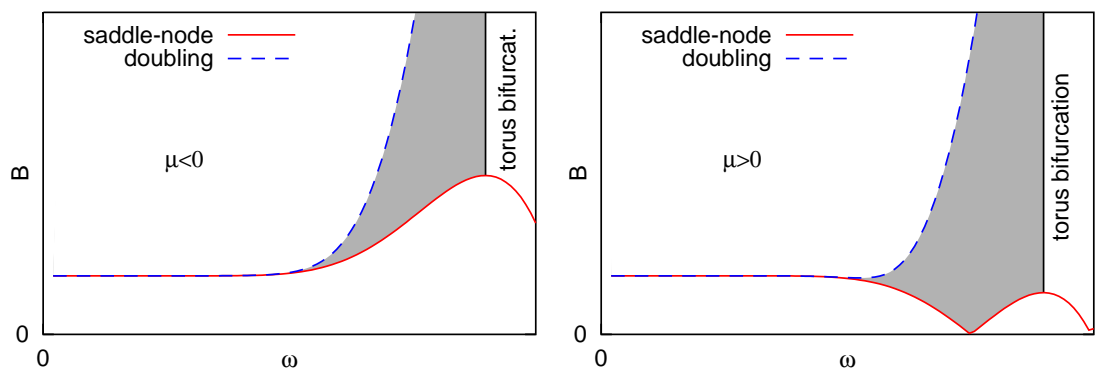

Figure 8: Bifurcation diagram for periodic $\varphi$-rotations. Gray regions: existence of stable periodic state. Left panel: $\mu<0$ (autonomous system is pre-homoclinic). Right panel: $\mu>0$ (post-homoclinic situation).

Summarizing we see that in both cases, for oscillations and rotations, periodic external modulation virtually does not induce phase locking at sufficiently low driving frequencies: the range of amplitude in which the locked periodic state is stable, is exponentially small.

\section{Spin-torque oscillator under periodic force: bifurcations of oscillatory states}

Let us apply results from the preceding section to the case of the spin-torque oscillator influenced by the periodic force. To this end, we consider periodic modulation of the current with frequency $\Omega$ : $I(t)=$ $I_{0}+\eta \cos \Omega t$.

According to the general theory, self-sustained oscillations of the STO should be entrained by the modulation, provided that $\Omega$ is sufficiently close to the frequency of the autonomous system; the larger the mismatch between the frequencies, the higher should be the minimal modulation amplitude which ensures entrainment.

Indeed, we observe entrainment for the values of $I_{0}$ both below and above the gluing bifurcation in the autonomous equations. In both cases, the 1:1 entrainment occurs in the stripe of the parameter plane bounded 

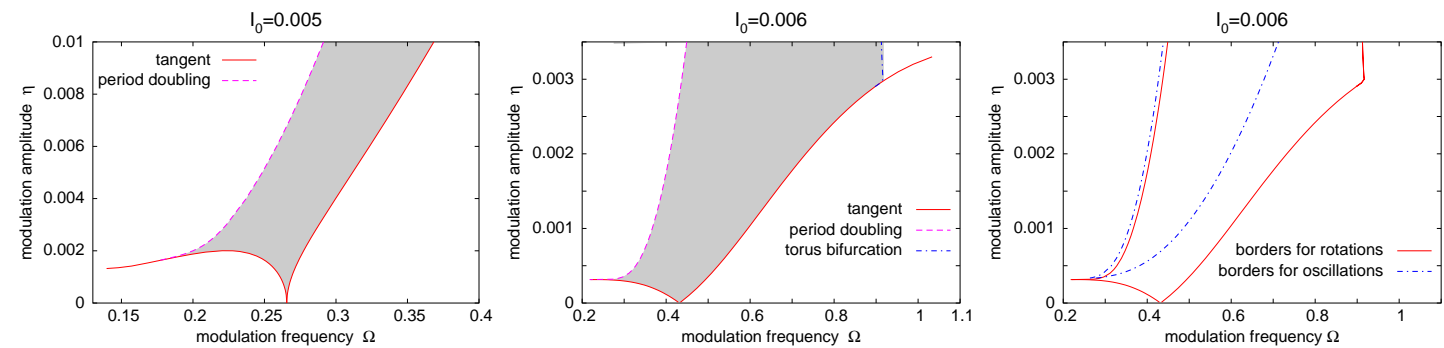

Figure 9: Main resonance regions on the parameter plane. Values of $I_{0}$ correspond to dynamics below (left panel) and above (center panel) the gluing bifurcation in the autonomous system. Right panel: Stability regions for periodic $\varphi$-oscillations and $\varphi$-rotations on the parameter plane.

by three curves: two curves of the tangent bifurcation and the curve of the period-doubling bifurcation, beyond which the trajectory in the phase space closes after two periods of modulation.

The shape of the resonant regions on the parameter plane bears apparent distinctions from the familiar wedge-like form of the Arnold tongues: the left borders are strongly deformed, the critical amplitude is a non-monotonic function of the modulation frequency. As predicted by analysis in the preceding section, at low modulation frequencies the interval of stability of the periodic state, is squeezed between the saddle-node bifurcation and the period-doubling bifurcation, and becomes extremely narrow. In accordance with the prediction, the curve of the torus (Neimark-Sacker) bifurcation where the limit cycle yields to quasiperiodicity, is nearly vertical: it can be encountered only in the rather narrow range of modulation frequencies. Therefore, in Fig. [9 this curve is shown only for $I_{0}=0.006$; the values of $\Omega$ used for plotting other bifurcation curves at $I_{0}=0.005$ in the left panel, are too low.

Furthermore, in large parts of the resonance regions the driven system is multistable: depending on the initial conditions, trajectories can converge to either of two oscillatory states. We demonstrate this effect in the right panel of Fig. 9, taking the value of $I$ above the threshold of the gluing bifurcation in the autonomous system. As seen in the plot, the main resonance region (bounded by red curves) which corresponds to the rotation of $\varphi$, largely overlaps with the region (bounded by the blue curves) in which $\varphi$ performs localized oscillations. The only difference is that at appropriate values of the modulation frequency, $\varphi$-rotations can be excited by arbitrarily weak driving, whereas the onset of $\varphi$-oscillations (absent in the autonomous system at this value of $I$ ) requires that the modulation amplitude exceeds a certain finite threshold.

Besides the simple periodic attractors, in the large regions of the parameter plane more complicated oscillations are observed: they include multi-turn limit cycles, quasiperiodic states as well as apparently chaotic attractors. Detailed investigation of transitions between those states lies outside the scope of the present paper; below we briefly describe their main types. Like simple limit cycles, the multi-turn ones can be roughly divided into $\varphi$-oscillatory and $\varphi$-rotating ones; in Fig. 10 we present a few characteristic phase portraits for periodic states with zero net rotation along $\varphi$; during such oscillations several cycles of clockwise rotations are followed by the same amount of counterclockwise ones.

Quasiperiodic and weakly chaotic states are exemplified in Fig. 5 which shows the projections of stroboscopic mappings. Outside the Arnold tongues, at low amplitudes of the driving force the motions are quasiperiodic; the mapping possesses two symmetric invariant curves which correspond to quasiperiodic rotations in two opposite directions. When the amplitude is increased, the curves approach each other and "recombine"; coarsely, transition to chaos occurs after this event.

Altogether, as follows from theoretical predictions [19], dynamics is quite rich, and the region where the STO just follows the external force occupies only a relatively small part of the parameter plane. 

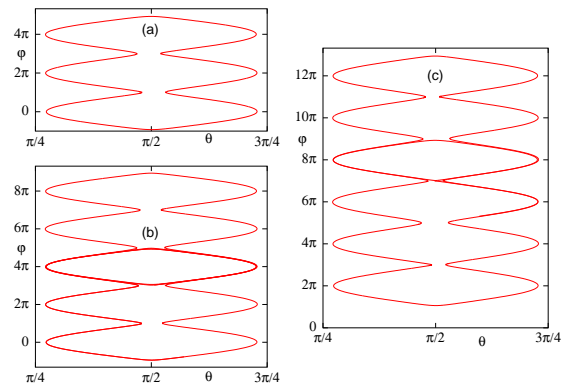

Figure 10: Projections of phase portraits for multi-turn periodic states. $I_{0}=0.006, \Omega=0.3$. (a): $\eta=0.002 ;$ (b): $\eta=0.015$; (c): $\eta=0.0012$.
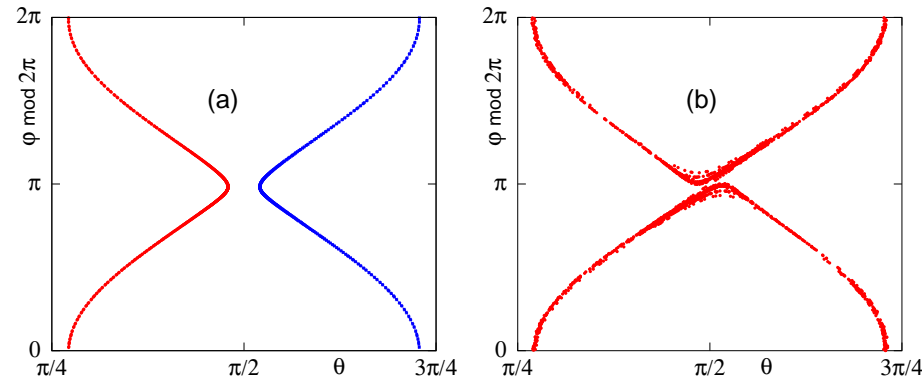

Figure 11: Stroboscopic mapping for quasiperiodic (a) and chaotic (b) oscillations. $I_{0}=0.006, \Omega=0.3$. (a): Two orbits at $\eta=0.0005$. Red dots: clockwise $\varphi$-rotation. Blued dots: counterclockwise $\varphi$-rotation. (b): $\eta=0.0035$. The single attracting orbit for which rotations in both directions alternate intermittently.

\section{Discussion}

\subsection{Summary of results}

We have characterized the typical features of entrainment by periodic external force for systems close to a homoclinic (gluing) bifurcation. General character of results allows to expect that similar deformation of Arnold tongues with such consequences as multistability and/or chaos takes place in many periodically forced systems: Unstable steady states play important dynamical role in a variety of problems of fluid mechanics, nonlinear optics, physical chemistry, etc., and in many documented situations evolution of stable periodic regimes leads through homoclinic bifurcations of those states. In the above example the leading eigenvalues of the saddle are real. Quite often the relevant steady states are of the saddle-focus type; dynamically, this brings in another oscillatory timescale which interacts with the existing ones, and makes the adequate description of entrainment much more involved.

We have considered the simple harmonic forcing; for a periodic force with many Fourier harmonics, the deformation of entrainment region would be more drastic. This, for example, should be the case for a spin-torque oscillator unilaterally influenced by another STO unit which, itself, is close to homoclinicity. When the interaction is bilateral (e.g. the STO are serially connected), robust synchronization of two units can be restricted to small regions of the parameter space.

\subsection{Sketch of synchronization diagram for two coupled STO}

As an illustration, we take two identical or weakly non-identical serially connected STO; details of the setup are described e.g. in [10]. Each oscillator is characterized by the angles $\theta_{i}$ and $\varphi_{i}(i=1,2)$ that, respectively, obey the first two equations of the system (6). The interaction occurs through the common current in the circuit, affected by variations of magnetoresistance of both oscillators. In the equations this 
is expressed in terms of the variable $u$ (rescaled voltage) governed by

$$
\ddot{u}=\Omega_{0}^{2}\left(\left(1-\frac{\varepsilon}{2}\left(\sin \theta_{1} \cos \varphi_{1}+\sin \theta_{2} \cos \varphi_{2}\right)\right)\left(1-\frac{\dot{u}}{\omega}\right)-u\right) .
$$

Thereby, intensity of interaction is regulated by the parameter $\varepsilon$. In Fig. 12 we sketch the state diagram obtained by variation of $\varepsilon$ and the difference between the individual values of the parameter $H_{d z}$, responsible for the magnitude of demagnetization field in the units.

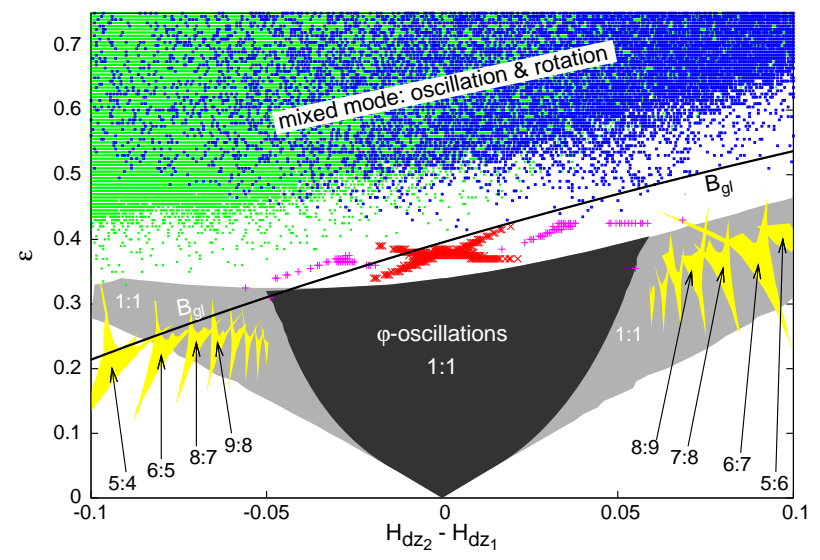

Figure 12: Sketch of the state diagram for two serially connected STO. Except for $\varepsilon$ and $H_{d z}$, the parameter values are taken from (7). Current $I$ is fixed at $0.0055 ; H_{d z_{1}}=1.6 ; \varepsilon$ and $H_{d z_{2}}$ are varied. White regions: quasiperiodic and chaotic asynchronous regimes with no stable periodic states. Black and gray regions: stable synchronous periodic $\varphi$-oscillations of both units; in the black region they are globally attracting. Yellow wedges: secondary resonance tongues with stable mode-locked states. Red crosses and magenta pluses: synchronous periodic states with, respectively, 2 and 4 turns in the phase space. Green and blue dots: coexisting mixed-mode states in which one unit performs periodic $\varphi$-oscillations whereas the other one rotates. Curve $B_{\mathrm{gl}}$ : gluing bifurcation for the isolated second STO.

Detailed analysis of bifurcations in the set of two coupled STO lies outside the scope of this paper and will be presented elsewhere. Here, we restrict ourselves to the most general features. The state diagram can hardly be directly compared to the treated above situation of periodic external driving, since the meaning of coordinates in Fig. 12 and e.g. in Fig. 9 is obviously different. This mostly concerns the ordinate of two diagrams. In contrast to the modulation amplitude $\eta$ that becomes relevant only in presence of forcing, the parameter $\varepsilon$, responsible for the coupling, enters the governing equations of the uncoupled STO oscillator as well: the corresponding term in the equation for $d u / d t$ describes "self-action" of the oscillator caused by temporal dependence of its magnetoresistance. Variation of $\varepsilon$, taken alone, can result in bifurcations of Eqs (6). As seen from Eq.(11), the Hopf bifurcation is $\varepsilon$-insensitive; in contrast, the gluing bifurcation depend on $\varepsilon$. In the range $1.5<H_{d z}<1.7$, corresponding to the diagram of Fig. 12, an uncoupled STO performs $\varphi$-oscillations at small values of $\varepsilon$, and $\varphi$-rotations at the higher values of that parameter; transition between these two types of motion is mediated by the gluing bifurcation. Close to that event, period of oscillations grows (recall Fig. 5b); thereby, unlike the modulation amplitude $\eta$, the parameter $\varepsilon$ influences the timescale of an uncoupled unit. In the case of external driving, the parameter plane is divided by the vertical line passing through the tip of the main resonance region: to the left from that line the uncoupled system is faster than the driving force, and in the right half-plane the opposite situation takes place. This clear dichotomy is absent in the case of two coupled units: at fixed values of $H_{d z_{1}}$ and $H_{d z_{2}}$ there exist ranges of $\varepsilon$ in which the frequency of oscillations in the isolated first STO exceeds the frequency of the second one, as well as ranges of $\varepsilon$ where the second oscillator is faster. Therefore a mapping between the regions of Fig. 12 and Fig. 9 is, to put it mildly, not straightforward.

At low values of $\varepsilon$, simple synchronous $\varphi$-oscillations of both units have been observed in parts of the parameter plane (black and gray regions in Fig. 12). However, they are globally attracting only at relatively 
small values of $\left|H_{d z_{1}}-H_{d z_{2}}\right|$; otherwise, they coexist with quasiperiodic or mode-locked motions, and their attraction basin shrinks when the difference between the values of $H_{d z}$ grows. This bistability provides a distinction from the conventional picture of synchronization in which quasiperiodic and periodic states do not coexist. On the upper boundary of the region of synchronous $\varphi$-oscillations, the Neimark-Sacker bifurcation takes place, and asynchronous quasiperiodic regimes replace periodic oscillations. Quasiperiodicity, in turn, yields to chaos; in certain regions of the plane we observe periodic windows where the units perform multiturn oscillations with zero net rotation of $\varphi$, akin to the orbits from Fig. 10.

At higher values of $\varepsilon$ the periodic mixed-mode states are present: one unit performs $\varphi$-oscillations whereas the other one synchronously rotates. In most of the cases, two such states coexist, albeit with different size of attraction basins: in one of them the unit with larger $H_{d z}$ oscillates and the other one rotates, in the second state the roles are interchanged. Two parameter regions with synchronous periodic attractors at the bottom and at the top of the plot are separated by the wide stripe with no stable periodic states. Remarkably, this stripe lies on both sides from the curve of the gluing bifurcation in the uncoupled unit. It should be noted that the latter curve does not denote a bifurcation in the coupled system: there, the saddle equilibrium has two-dimensional unstable manifold and cannot directly participate in bifurcations of stable periodic orbits. Nevertheless, in this region of the parameter plane the characteristic times of two oscillators lie sufficiently far apart, and sustainment of synchronous states becomes hardly possible, both for identical $\left(H_{d z_{1}}=H_{d z_{2}}\right)$ and non-identical STO oscillators. This confirms our conclusion that proximity of separate units to the gluing bifurcation hampers synchronization.

\subsection{Relevance for experimental observations}

The last remark concerns a possible comparison of the above results to experimental observations. It should be kept in mind, that in experiments at the nanoscales it is difficult to separate purely deterministic features from noisy and often non-stationary background. The role of fluctuations is not reduced to additive noise: e.g. the parameter drift can modulate intrinsic frequencies, and thereby cause and/or interrupt temporary epochs of synchrony. The appropriate theoretical description should include both the dynamical and the stochastic aspects.

\section{Acknowledgement}

Our research was supported by the Research Grant PI 220/17-1 of the DFG.

\section{References}

[1] I. I. Blekhman, Synchronization in science and technology, ASME Press Translations. 1988.

[2] S. Strogatz, Nonlinear dynamics and chaos: with applications to physics, biology, chemistry, and engineering. Perseus Books, 1994.

[3] J. A. Katine, F. J. Albert, R. A. Buhrman, E. B. Myers, and D. C. Ralph, Current-driven magnetization reversal and spin-wave excitations in $\mathrm{Co} / \mathrm{Cu} / \mathrm{Co}$ Pillars, Phys. Rev. Lett. 84, 3149 (2000).

[4] G. Finocchio, M. Carpentieri, A. Giordano, and B. Azzerboni, Non-Adlerian phase slip and nonstationary synchronization of spin-torque oscillators to a microwave source, Phys. Rev. B 86, 014438 (2012).

[5] M. Quinsat, V. Tiberkevich, D. Gusakova, A. Slavin, J. F. Sierra, U. Ebels, L. D. Buda-Prejbeanu, B. Dieny, M.-C. Cyrille, A. Zelster, and J. A. Katine, Linewidth of higher harmonics in a nonisochronous auto-oscillator: Application to spin-torque nano-oscillators, Phys. Rev. B 86, 104418 (2012).

[6] Y. Zhou, V. Tiberkevich, G. Consolo, E. Iacocca, B. Azzerboni, A. Slavin, and J. Åkerman, Oscillatory transient regime in the forced dynamics of a nonlinear auto oscillator, Phys. Rev. B 82, 012408 (2010).

[7] A. Slavin, and V. Tiberkevich, Nonlinear auto-oscillator theory of microwave generation by spin-polarized current, IEEE Trans. Magn. 45, 1875 (2009).

[8] D. Li, Y. Zhou, B. Hu, C. Zhou, Coupled perturbed heteroclinic cycles: synchronization and dynamical behaviors of spin-torque oscillators, Phys. Rev. B 84, 104414 (2011).

[9] D. Li, Y. Zhou, B. Hu, J. Akerman, C. Zhou, Multiple synchronization attractors of serially connected spin-torque nanooscillators, Phys. Rev. B 86, 014418 (2012).

[10] A. Pikovsky, Robust synchronization of spin-torque oscillators with an LCR load, Phys. Rev. E 88, 032812 (2013).

[11] J. M. Gambaudo, P. Glendinning and C. Tresser, The gluing bifurcation: I. Symbolic dynamics of closed curves, Nonlinearity 1, 203 (1988). 
[12] J. Turtle, K. Beauvais, R. Shaffer, A. Palacios, V. In, T. Emery, and P. Longhin, Gluing bifurcations in coupled spin torque nano-oscillators, J. Appl. Phys. 113, 114901 (2013).

[13] D. Li, Y. Zhou, C. Zhou, and B. Hu, Global attractors and the difficulty of synchronizing serial spin-torque oscillators, Phys. Rev. B 82, 140407 (2010).

[14] J. C. Slonczewski, Current-driven excitation of magnetic multilayers, J. Magn. Magn. Mater, 159, L1 (1996).

[15] J. Grollier, V. Cros, and A. Fert, Synchronization of spin-transfer oscillators driven by stimulated microwave currents, Phys. Rev. B 73, 060409 (2006).

[16] L. P. Shilnikov, Some cases of generation of periodic motions from singular trajectories, Mat. Sbornik 61 (103), 443 (1963).

[17] L. P. Shilnikov, On the generation of a periodic motion from a trajectory doubly asymptotic to an equilibrium state of saddle type, Math. USSR-Sb. 6, 428 (1968).

[18] L. P. Shilnikov, A. Shilnikov, D. Turaev and L. Chua, Methods of Qualitative Theory in Nonlinear Dynamics. Part II. World Scientific, 2001.

[19] V. S. Afraimovich, and L.-P. Shilnikov, Invariant two-dimensional tori, their breakdown and stochasticity, Amer. Math. Soc. Transl. 149, 201-212 (1991).

[20] D. V. Lyubimov, and S. L. Byelousova, Onset of homoclinic chaos due to degeneracy in the spectrum of the saddle. Physica D 62, 317-322 (1993).

[21] M. A. Zaks, E.-H. Park, and J. Kurths, On phase synchronization by periodic force in chaotic oscillators with saddle equilibria. Int. Journ. Bifurcations and Chaos 10, 2649-2667 (2000). 